\title{
CaRIÓtIPO EM LEUCEMIA MIELÓIDE AGUDA: IMPORTÂNCIA E TIPO DE ALTERAĢÃO EM 30 PACIENTES AO DIAGNóstico
}

\author{
Luís Arthur flores Pelloso, Maria de lourdes lopes Ferrari Chauffaille*, fabiana Sinnot Ghaname, \\ Minoko Yamamoto, Daniella Márcia Maranhão Bahia e José Kerbauy \\ Trabalho realizado na Disciplina de Hematologia e Hemoterapia da \\ Universidade Federal de São Paulo - UNIFESP - EPM, São Paulo, SP
}

Resumo - Objetivo. Análise sob o ponto de vista citogenético e clínico de pacientes com LMA.

Métodos. Foram estudados 30 pacientes, sendo 16 homens e 14 mulheres. A idade variou de 19 a 84 anos. 0 diagnóstico baseou-se na classificação OMS, imunofenotipagem e citogenética clássica por banda G. Foram tratados com o protocolo daunorrubicina e citarabina $(3+7)$, com adição de ATRA na Leucemia Promielocítica Aguda.

Resultados. A taxa de sucesso de cariótipo foi $84 \%$. De acordo com o cariótipo, os pacientes foram divididos em quatro grupos: prognóstico favorável (PF) $(6)(t(8 ; 2 I), t(I 5 ; 17))$; intermediário (PI) (7) (quatro casos com cariótipo normal, $+8, \mathrm{t}(\mathrm{I} ; 2)$ e del 18(q)); desfavorável (PD) (10) e três casos eram LMA secundária (dois evoluídos de síndrome mielodisplásica e uma apresentação de LMC em crise blástica. No grupo de PF, a idade mediana foi 23 e no PD 60 anos $(p<0,003)$. No grupo PF, $5 / 6$ pacientes $(83 \%)$ entraram em remissão completa (RC), no $\mathrm{PI}$ I/7 (20\%) e no PD I/8 (I2,5\%). Houve tendência à leucometria mais elevada no grupo de PD.

ConcLusöEs. A porcentagem de cariótipo alterado em LMA (80\%) está dentro dos valores relatados pela literatura (65\%-95\%). Ficou nítida a diferença entre a taxa de $R C$ do grupo $P F$ versus $P D$, confirmando a importância do cariótipo na diferenciação dos grupos de risco.

\section{INTRODUÇÃO}

A Leucemia Mielóide Aguda (LMA) se caracteriza pela proliferação clonal de células blásticas derivadas da célula tronco hematopoética com conseqüente substituição do tecido normal. Não se conhece sua etiologia, porém algumas lesões genéticas estão implicadas no seu desenvolvimento'.

Aimunofenotipagem permite reconhecer o clone anormal, definir a linhagem, o estágio de diferenciação, características prognósticas e fenótipos aberrantes para monitorar a doença residual mínima ${ }^{2,3}$.

A citogenética, no diagnóstico da LMA, é um marcador prognóstico bem definido ${ }^{4-5}$. Anormalidades cromossômicas caracterizadas por translocações balanceadas, perda e ganho de cromossomos são peculiares às leucemias e encontradas em mais de $65 \%$ dos casos'. É considerado pelo grupo SWOG como cariótipo favorável: inv $(16) / \mathrm{t}(16 ; 16) / \operatorname{del}(16 q)$, $\mathfrak{t}(|5 ;| 7)$ com ou sem alterações secundárias,

\footnotetext{
*Correspondência:

Rua Botucatu 740 - $3^{\circ}$ andar - 04023-900 São Paulo - Capital

Telefone: (11) 5576-4240 - FAX: 5571-8806 e-mail: chauffaill@hemato.epm.br
}

$\mathrm{t}(8 ; 2 \mathrm{I})$ sem del( $9 \mathrm{q})$ ou cariótipo complexo; como intermediário: cariótipo normal, +8 , $+6,-Y$, del( $(2 p)$ enquanto como cariótipo desfavorável alterações envolvendo os cromossomos 3, 9, II, 20, 21, del (5q), -5, del(7q), -7 e cariótipos complexos ${ }^{5}$.

O objetivo do presente trabalho é analisar pacientes com LMA consecutivamente admitidos na UNIFESP-EPM, sob o ponto citogenético eatravés de dados clínico-laboratoriais, como sexo, idade, nível de hemoglobina, leucometria e taxa de remissão completa.

\section{Métodos}

Foram avaliados 30 pacientes com diagnóstico de LMA consecutivamente admitidos no Hospital São Paulo, UNIFESP- EPM entre janeiro de 2000 e maio de 200I. Havia 16 homens e 14 mulheres com mediana de idade de 50,5 anos (variando de 19 a 84 anos).

O diagnóstico de LMA baseou-se na contagem de células blásticas dos esfregaços de medula óssea com número igual ou superior a $20 \%$, juntamente com a imunofenotipagem ${ }^{6-7}$.

A análise citomorfológica foi feita em esfregaços de medula óssea corados com May-Grünwald e Giemsa e classificados de acordo com os critérios propostos pelo grupo
OMS com o uso de reações citoquímicas (peroxidase, Sudan-Black B, alfa esterase e inibição com fluoreto de sódio) ${ }^{6-12}$.

A análise imunofenotípica foi feita tanto em blastos de medula óssea (MO) como de sangue periférico. Foi usado o seguinte painel de anticorpos monoclonais (marcação direta em tripla coloração, ficoeritrina( $\mathrm{PE}) /$ isotiociananto de fluorescína (FITC)/ peridina clorofila (PerCP): CDI0; CDIIc; CDI3; CD I4; CD 19; CD33; CD34; HLADR; CD2; CD4; CDI5; CD65; CDII7; CD7; CD38; CD4I; CD56 (Becton Dickson, Immunotech, DAKO). A aquisição e análise dos dados foram realizadas em citômetro de fluxo (FACScalibur Becton Dickinson Imunocytometry Systems San Jose California, CA) e a positividade para determinado antígeno foi definida quando sua expressão estava presente em $20 \%$ ou mais das células leucêmicas ${ }^{3}$.

O material destinado à citogenética clássica foi colocado em cultura de curta duração da forma habitual ${ }^{13}$. Foram realizadas duas culturas com RPMI, $7 \mathrm{~mL}$, soro fetal bovino, $3 \mathrm{~mL}$ e $100 \mathrm{~mL}$ de Lglutamina. Este material foi mantido em estufa a $37^{\circ} \mathrm{C}$ durante 24 horas e acres- 
Gráfico I - Distribuição dos pacientes com LMA por idade e cariótipo

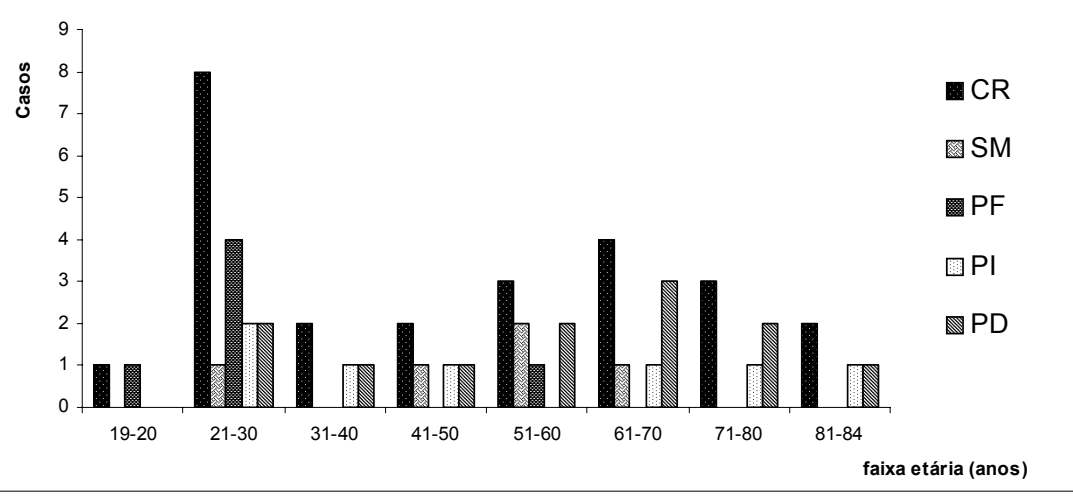

CR: cariótipo com resultado, SIM: cariótipo sem resultado, PF: cariótipo de prognóstico favorável: $t(8 ; 21), t(\mid 5 ; 17)$, PI: cariótipo de prognóstico intermediário: cariótipo normal, $+8, \mathrm{t}(\mid ; 2)$ e del (I8p), e PD: cariótipo de prognóstico desfavorável del (5q)/-5, del(7q)/-7, abn3q, + II, t(9;22) e cariótipos complexos

centados $50 \mathrm{~mL}$ de colchinina por $30 \mathrm{mi}$ nutos. Em seguida, o material foi exposto à solução hipotônica ( $\mathrm{KCL} 0,075 \mathrm{~mol} / \mathrm{L})$ seguida de fixação com solução de ácido acético e metanol (3:I) por três vezes. Foram feitas lâminas destinadas à análise cromossômica pela banda $G$ pela técnica de tripsina-Giemsa (GTG), sendo posteriormente analisadas 20 metáfases e capturadas as imagens em sistema computadorizado com software Cytovision ${ }^{13}$. As anormalidades cromossômicas foram descritas de acordo com a ISCN ${ }^{14}$.

Os pacientes foram tratados com a quimioterapia de indução: daunorrubicina $45 \mathrm{mg} / \mathrm{m}^{2} / \mathrm{d}$ por três dias e citarabina $100 \mathrm{mg} /$ $\mathrm{m}^{2} / \mathrm{d}$ por sete dias em infusão contínua. Os casos de leucemia promielocítica aguda receberam adicionalmente ácido trans-retinóico (ATRA) na dose de $45 \mathrm{mg} / \mathrm{m}^{2} /$ dia até a remissão completa.

A remissão completa $(\mathrm{RC})$ foi considerada quando a medula era normocelular ou hipercelular, mas com menos que $5 \%$ de blastos, sem bastão de Auer e na presença dos seguintes parâmetros hematológicos: granulócitos em número igual ou superior a I, $5 \times 10^{9} / \mathrm{L}$; plaquetas igual ou superior a $100 \times 10^{9} / \mathrm{L}$ e $\mathrm{Hb}>10 \mathrm{~g} / \mathrm{dL}^{15}$. Os pacientes que apresentaram entre $5 \%$ e $25 \%$ de blastos foram considerados em remissão parcial (RP) e aqueles com mais de $25 \%$ de blastos como ausência de remissão (NR) ${ }^{15}$.

\section{Resultados}

A Tabela I mostra as características analisadas: idade, sexo, subtipo OMS, cariótipo, valores de hemoglobina, leucometria ao diagnóstico e a aquisição de remissão completa. $O$ Gráfico I mostra a distribuição dos pacientes conforme idade e resultado do cariótipo.

Os pacientes foram separados em quatro grupos conforme os resultados do cariótipo em: a) favorável(PF): $t(8 ; 2 I), t(|5 ;| 7) ; b)$ intermediário(PI): cariótipo normal, +8 , $\mathrm{t}(\mathrm{I} ; 2)$, del( (18q); c) desfavorável (PD):del(5q)/ $-5, \operatorname{del}(7 \mathrm{q}) /-7, \operatorname{abn}(3 \mathrm{q}),+\mathrm{Ilq}, \mathfrak{t}(9 ; 22)$ e cariótipos complexos ed) LMAsecundária (LS) (Gráfico I).

Dos 30 pacientes, obteve-se resultado de cariótipo em 25 (84\%). Em cinco casos não se obtiveram metáfases. Com relação à idade, $63,6 \%$ dos pacientes com mais de 50 anos pertenciam ao grupo de cariótipo desfavorável e $83,4 \%$ dos pacientes do grupo de cariótipo favorável tinham idade até 50 anos.

No grupo de cariótipo favorável todos os seis pacientes receberam tratamento adequado, dos quais cinco alcançaram RC (83\%). Houve uma morte na indução devido à Síndrome do ácido trans-retinóico.

Oito de dez pacientes do grupo de cariótipo desfavorável $(80 \%)$ receberam terapia de indução, tendo um atingido a RC (I2,5\%), dois foram a óbito na fase de indução e 37,5\% $(n=3)$ ficaram com LMA refratária.

Nogrupo de prognóstico intermediárioe com cariótipo normal, cinco dentre sete pacientes $(71,4 \%)$ receberam indução. Os demais não apresentavam condições clínicas para tratamento e um caso (25\%) foi a óbito na fase de indução. Dos cinco pacientes, um caso entrou em remissão completa (20\%).

Três casos não eram LMA primária (casos I5, 25 e 28 - Tabela I). O primeiro (caso I5) tratava-se de uma rara apresentação inicial de crise blástica mielóide de Leucemia Mielóide Crônica. Os casos 25 e 28 tinham diagnóstico prévio de síndrome mielodisplásica em outro serviço e a LMA era evolução da doença (Tabela 2). Nesse grupo de LMA secundária, a idade mediana foi a mais alta, 70 anos, e somente um paciente recebeu indução, mas não entrou em remissão completa. Os outros apresentavam condições clínicas precárias e idade elevada e por isso receberam tratamento de suporte.

Não houve diferença na taxa de hemoglobina entre os grupos e a leucometria apresentou tendência a ser mais alta no grupo de cariótipo desfavorável. Houve diferença estatisticamente significante $(p=0.003)$ na idade entre os grupos, sendo a mediana da idade do grupo desfavorável maior que a do grupo favorável (Tabela 2).

\section{Discussão}

De acordo com os estudos do SWOG (Southwest Oncology Group) e MRC (Medical Research Council), os pacientes são estratificados conforme a alteração citogenética específica em três grupos: favorável, intermediário e mau prognóstico, com nítidas diferenças na sobrevida ${ }^{4,5,16}$. No grupo SWOG, a sobrevida projetada para oito anos para o grupo de cariótipo favorável é de $55 \%$, para o grupo intermediário $38 \%$ e para o grupo de prognóstico desfavorável, II\% com $p<.000 \mathrm{I}^{16}$. No presente trabalho, $83 \%$ dos pacientes do grupo de cariótipo favorável alcançou $R C$ versus 12,5\% do grupo de cariótipo desfavorável.

Embora a freqüência global de anormalidades cromossômicas não pareça estar associada diretamente à idade, algumas alterações citogenéticas são mais freqüentes em determinadas faixas etárias ${ }^{17}$. Aneuploidia parece ser um evento muito mais freqüente em idosos e anormalidades como monossomia ou deleção envolvendo os cromossomos 5 e 7 representam $26 \%$ das alterações encontradas em 


\begin{tabular}{|c|c|c|c|c|c|c|c|c|}
\hline $\mathbf{N}$ & $\begin{array}{l}\text { Idade } \\
\text { (anos) }\end{array}$ & Sexo & $\begin{array}{l}\text { Classificação } \\
\text { OMS }\end{array}$ & Cariótipo por banda G & $\begin{array}{l}\text { Hemoglobina } \\
5,0-13,9 \mathrm{~g} / \mathrm{dL})\end{array}$ & $\begin{array}{c}\text { Leucometria } \\
\left(600-390.000 / \mathrm{mm}^{3}\right)\end{array}$ & $\mathrm{RCl}$ & RC2 \\
\hline । & 59 & $\mathrm{~F}$ & LMANCMo & $47, X X,+11[5] / 46, X X[10]$ & 6,1 & 43.000 & Não & \\
\hline 2 & 61 & $\mathrm{~F}$ & LMANCMo & $46, X X, t(9 ; 22)(q 34.1 ; q \mid I . I)[15]$ & 5,0 & 22.000 & Sim & \\
\hline 3 & 50 & M & LMADispS/SMD & $45, \mathrm{XY},([3 ; 3)(\mathrm{q} 21 ; q 27), t(5 ; 12)(\mathrm{q} 31 ; q 242.1),-7, \mathrm{add}[\mathrm{II}](\mathrm{p} \mid 5)[20]$ & ] 9,8 & 1.200 & Não & \\
\hline 4 & 46 & $\mathrm{~F}$ & $L A B$ & $46, X X, t(9 ; 22)(q 34 . I ; q \mid I)[15]$ & 11,5 & 42.600 & Não & \\
\hline 5 & 23 & $\mathrm{~F}$ & LMANCS/Mat & $46, X X, \operatorname{del}(18)(q 21)[15]$ & 5,2 & 4.000 & Sim & \\
\hline 6 & 72 & $\mathrm{~F}$ & LMANCMo & $46, X X, t(3 ; 3)(q 21 ; q 26)[20]$ & 8,6 & 97.160 & Não & \\
\hline 7 & 29 & M & $\operatorname{LMAt}(8 ; 21)$ & $46, \mathrm{XY}, \mathrm{t}(8 ; 21)(\mathrm{q} 22 ; \mathrm{q} 22)[20]$ & 5,9 & 42.000 & Sim & \\
\hline 8 & 28 & M & LMANCMielomono & $\mathrm{S} /$ mitose & 13,9 & 88.000 & Sim & \\
\hline 9 & 58 & $M$ & LMANCMD & S/mitose & 8,7 & 255.000 & Não & \\
\hline 10 & 51 & $\mathrm{~F}$ & $\operatorname{LMAt}(|5 ;| 7)$ & $46, X X, t(15 ; \mid 7)(q 22 ; q \mid I)[15]$ & 10 & 117.000 & Não & \\
\hline II & 44 & $M$ & LMANCMo & $46, X Y, t(1 ; 2)(p 31 ; q 34)[20]$ & 6,5 & 306.000 & Não & \\
\hline 12 & 22 & $M$ & LMANCS/Mat & $47, \mathrm{XY},+8[15] / 46, \mathrm{XY}[4]$ & 9,7 & 263.000 & Não & Sim \\
\hline 13 & 31 & $M$ & LMANCC/Mat & $46, X Y[20]$ & 6,0 & 600 & Não & não \\
\hline 14 & 23 & M & $\operatorname{LMAt}(8 ; 21)$ & $46, \mathrm{XY}, \mathrm{t}(8 ; 21)(\mathrm{q} 22 ; \mathrm{q} 22)[15]$ & 8,1 & 6.000 & Sim & \\
\hline 15 & 25 & $\mathrm{~F}$ & LMANCMega* & 46,XX,t(9,22)(q34.।;;q I.2)[20] & 10,3 & 10.300 & Não & \\
\hline 16 & 23 & $M$ & $\operatorname{LMAt}(8 ; 21)$ & $46, \mathrm{XY}, \mathrm{t}(8 ; 21)(\mathrm{q} 22 ; \mathrm{q} 22)[15]$ & 6,5 & 7.200 & Sim & \\
\hline 17 & 19 & $\mathrm{~F}$ & LMA t $(15 ; 17)$ & $46, X X, t(\mid 15 ; 17)(q 22 ; q \mid I)[20]$ & 8,1 & 1.700 & Sim & \\
\hline 18 & 33 & M & LMANCMega & $46, X Y, t(4 ; \mid 2)(q|| ; p \mid 3)[20]$ & 10,3 & 60.600 & não & \\
\hline 19 & 67 & $\mathrm{~F}$ & LMANCC/Mat & $46, X X[20]$ & 6,6 & 2.300 & não & \\
\hline 20 & 84 & M & LMADisp C/SMD & $46, X Y[20]$ & 8,5 & 4.200 & Não & \\
\hline 21 & 54 & $\mathrm{~F}$ & LMANCC/Mat & $47, \mathrm{XY},+1 \mathrm{I}[10] / 46, \mathrm{XX}[10]$ & 9,2 & 390.000 & Não & \\
\hline 22 & 21 & M & $\operatorname{LMA} t(15 ; 17)$ & $46, X Y, t(15 ; \mid 7)(q 22 ; q 1))[20]$ & 12,9 & 10.200 & Sim & \\
\hline 23 & 49 & M & LMANCMD & S/mitose & 7,5 & 1.000 & Sim & \\
\hline 24 & 78 & M & LMADispS/SMD & $45, X Y,-7[16] / 46, X Y[4]$ & 6,4 & 15.000 & Não & \\
\hline 25 & 70 & M & LMADisp C/SMD & S/mitose & 9,3 & 900 & Não & \\
\hline 26 & 68 & $\mathrm{~F}$ & LMADispS/SMD & $\begin{array}{l}47, X X,+8[1] / 47, X X, \operatorname{del}(5)(q),(q 34), \\
\operatorname{del}(7)(q 32),+8[2] / 49, X X, \operatorname{del}(7)(q 32), \\
+8,+11,+18[1]\end{array}$ & 11,5 & 1.700 & Não & \\
\hline 27 & 55 & M & LMADisp S/SMD & S/mitose & 9,6 & 4.080 & Sim & \\
\hline 28 & 81 & $\mathrm{~F}$ & LMADisp C/SMD & $46, X X[20]$ & 8,7 & 71.000 & Não & \\
\hline 29 & 65 & $\mathrm{~F}$ & LMADispS/SMD & $47, X X, \operatorname{del}(7)(q 31),+8[3] / 46, X X[17]$ & 6,0 & 90.000 & Não & \\
\hline 30 & 75 & $\mathrm{~F}$ & LMA NCC/Mat & $46, X X,[20]$ & 6,5 & 2.100 & não & \\
\hline
\end{tabular}

N: pacientes admitidos consecutivamente, idade em anos, sexo F:feminino, M: masculino, Classificação OMS: Organização Mundial da Saúde (Harris et al 1999). Cariótipo, Hemoglobina em g/dL, leucometria em mm³ ${ }^{3}$ RCI: primeira remissão completa e RC2: remissão completa após reindução. LMANCMo: LMA não categorizada Monocítica, LMA Disp S/SMD LMA com displasia multilinhagem sem SMD prévia, LAB: Leucemia Aguda Bifenotípica, LMA NCS/Mat: LMA não categorizada sem maturação, LMA t(8;2I): LMA com alteração citogenética recorrente t(8;2I), LMANC Mielomono: LMA não categorizada mielo monocítica, LMANCMD: LMA não categorizada minimamente diferenciada LMAt(15;17):LMA com alteração citogenética recorrentet(15;17), LMANCC/Mat:LMAnão categorizada com maturação, LMANCMega:LMAnão categorizada megacarioćitica, LMADisp C/SMD:LMA com displasiamultilihagem sem SMD prévia * $O$ caso I 5 tratava-se inicialmente de LMAMegacariocítica, porém a citogenética demonstrou t $(9 ; 22)$ e o RT-PCR p2I0, concluindo que se tratava de apresentação inicial de crise blástica de LMC ${ }^{19}$.

idosos $^{18}$. Neste estudo, os pacientes com cariótipo favorável apresentaram mediana da idade menor em relação ao grupo de cariótipo desfavorável (23 versus 60 anos). As trans- locações consideradas de bom prognóstico como $t(8 ; 2 I), t(15 ; \mid 7)$ e inv $(16)$ são muito mais freqüentes em indivíduos jovens, ainda que possam ocorrer em grupos etários mais avançados e, quando o fazem, são em baixa porcentagem (7\%), conforme demonstrado por Grimwade et al ${ }^{18}$. Já os pacientes com cariótipos desfavoráveis apresentaram verda- 


\begin{tabular}{|c|c|c|c|c|c|}
\hline & & & & IMA Socundária & \\
\hline Cariótipo & Favorável & Intermediário & Desfavorável & & Sem mitoses \\
\hline $\operatorname{Casos}(n)$ & 6 & 7 & 10 & 3 & 4 \\
\hline RelaçãoH:M & $2: 1$ & $1,33: 1$ & $0,42: 1$ & $0,5: 1$ & 4:0 \\
\hline Idade(anos) & 23 & 44 & 60 & 70 & 52 \\
\hline Hemoglobina g/dL & 8,1 & 6,5 & 8,9 & 9,3 & 9,15 \\
\hline Leucometriamm ${ }^{3}$ & 8.700 & 4.000 & 42.800 & 10.300 & 46.040 \\
\hline Quimioterapiaindução & $6(100 \%)$ & $5(71,4 \%)$ & $8(80 \%)$ & $\mathrm{I}(33,3 \%)$ & $4(100 \%)$ \\
\hline Remissão completa & $5(83 \%)$ & I(20\%) & $\mathrm{I}(12,5 \%)$ & 0 & 1(25\%) \\
\hline Remissãoparcial & 0 & $2(40 \%)$ & $\mathrm{I}(12,5 \%)$ & l(100\%) & $\mathrm{I}(25 \%)$ \\
\hline Morteindução & $\mid(\mid 6,6 \%)$ & I(20\%) & $2(25 \%)$ & 0 & I(25\%) \\
\hline LMArefratária & 0 & $\mathrm{I}(20 \%)$ & $5(62,5 \%)$ & 0 & I(25\%) \\
\hline
\end{tabular}

Casos sem mitoses (8,9, 23 e27),cariótipo favorável ( $\mathrm{t}(8 ; 21)$ et $(15 ; 17)$ (casos 7,10,14,16,17 e22), intermediário(normal, +8et(1;2))(casos5,12,13, 18, 19, 20 e 30),

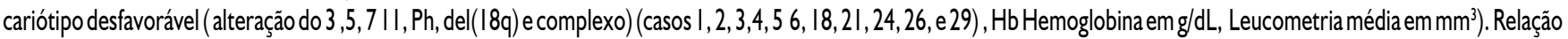
M:Fmasculino:feminino. Aidade, taxa de hemglobina ealeucometria foram calculdadas pela mediana. Variaçãodaidadenos grupos: sem mitose (28-58 anos), favorável(19-5l anos), intermediário (22-84 anos) edesfavorável (23-78anos). Houvediferença estatisticamentesignificante entreaidade do grupo de cariótipo favorável edesfavorável ( $\mathrm{p}=0,003)$.Variaçãoda hemoglobina nos diferentes grupos: sem mitose $(7,5-13,9 \mathrm{~g} / \mathrm{dL})$, favorável $(5,9-12,9 \mathrm{~g} / \mathrm{dL})$, intermediário $(5,2-10,3 \mathrm{~g} / \mathrm{dl})$, edesfavorável(5-II,5g/dL).VVariaçãodaleucometrianos grupos: semmitose $\left(1.000-255.000 / \mathrm{mm}^{3}\right)$, favorável $\left(1.700-117-000 / \mathrm{mm}^{3}\right)$, intermediário $\left(600-360.000 / \mathrm{mm}^{3}\right)$ edesfavorável $\left(1.200-390.000 / \mathrm{mm}^{3}\right)$.

deira miscelânea de alterações, a saber: dois com + I I ( l e2I), três envolvendo deleção ou monossomia do cromossomo 7 ( 24,29 e 3 sendo este último complexo), dois casos envolvendo o cromossomo 3 ( 3 e 6), dois com cromossomo Philadelphia ( 2 e 4 ), um envolvendo os cromosomos 5 e 7 (caso 26 com cariótipo complexo e alteração do 5 e 7q) (Tabela I). Indivíduos idosos representam um grupo significativo e importante de pacientes com LMA (I7-I8), e embora 55\% das LMAs ocorram em idosos, apenas $10 \%$ desses pacientes participam de ensaios clínicos ${ }^{17} \mathrm{e}$ a sobrevida global em cinco anos é de $20 \%{ }^{18}$. Uma série de fatores está implicada no padrão de resposta e sobrevida neste grupo, a saber: perfil de alterações citogenéticas desfavoráveis, superexpressão de fenótipo de multirresistência a drogas (MDR-I, LRPeMRP), maior freqüência de LMA secundária à síndrome mielodisplásica, mieloproliferativa ou a tratamento prévio, menor tolerância dos sistemas cardiovascular e renal-metabólico, presença de co-morbidades severas e menor nível de desempenho $0^{17-18}$. Neste trabalho, $23,3 \%$ dos pacientes apresentavam mais de $6 \mathrm{I}$ anos, e quando se diminui o nível de corte para $5 \mathrm{I}$ anos, $33,3 \%$ encontram-se acima desta faixa etária. Dos pacientes idosos analisados no presente estudo, $45 \%$ apresentavam alterações citogenéticas desfavoráveis e nenhum apresentou cariótipo de bom prognóstico.

As alterações numéricas são freqüentemente encontradas em LMA sendo a +8 relatada em $9 \%$ dos casos, -7 em $9 \%$ dos casos e $+1 \mathrm{l}$ em $0,7 \%$ dos casos $(16,19)$. A monossomia 7 , de acordo com Dastugue et a ${ }^{20} \mathrm{e}$ Joventino et al ${ }^{21}$, tem cerca de $82 \%$ de RC com sobrevida global de $17 \%$ em três anos e $0 \%$ em cinco anos. $A+8$ apresenta taxa de $R C$ de $66 \%$ com sobrevida de cinco anos de $27 \%{ }^{20}$. Nosso trabalho encontrou +8 em três casos (10\%) (12, 26 e 29$)$, sendo nos dois últimos como alteração adicional as outras alterações. A-7 foi encontrada em um paciente $(0,33 \%)^{24}$ e a + II foi encontrada em três $\operatorname{casos}(10 \%)$.

A presença de cariótipos infreqüentes, tanto anormalidades estruturais como numéricas, é uma peculiaridade citogenética vista em torno de $36 \%$ a $37 \%$ dos casos de LMA ${ }^{1,5}$. Estas alterações são geralmente consideradas de prognóstico desconhecido, mesmo porque são raras e é difícil projetar resposta e sobrevida. Nesta análise foram encontradas três alterações cromossômicas bastante infreqüentes: dois casos (5 e I I) ficaram alocados no grupo intermediário e um caso (paciente 18) no grupo de mau prognóstico. Esse último tem história já documentada com o caso da $\mathrm{t}(4 ; \mid 2)$, que se apresentou com comportamento clínico bastante agresssivo sugerindo que a origem da célula leucêmica fosse imatu$\mathrm{ra}^{22-24}$. O caso 5 apresentava del( $(18 \mathrm{q})$ e alcançou RC após indução convencional, porém evoluiu a óbito por fungemia e o caso II com $\mathfrak{t}(I ; 2)$ apresentou remissão completa com normalização citogenética (46,XY[20]) e recaída medular com infiltração de pele 18 meses após e com o mesmo cariótipo do diagnóstico.

Outro achado relativamente raro éa presença de cromossomo Philadelphia ${ }^{19}$. Encontramos dois pacientes (I8, I\% do grupo de cariótipo desfavorável), mas nenhum deles apresentava esplenomegalia, leucocitose com desvio à esquerda, eosinofilia, basofilia ou fibrose medular para inferir que pudessem ser crise blástica de Leucemia Mielóide Crônica (LMC) e, diferentemente do caso 15, que apresentava leucocitose e desvio à esquerda, fibrose medular grau III e rearranjo gênico $B C R / A B L \mathrm{p} 2 \mathrm{I0}$ e, portanto considerado como crise blástica de LMC. A presença do Philadelphia confere prognóstico reservado com taxa da RC média de $55 \%$, duração média de RC de 17 meses e sobrevida projetada em cinco anos de $0 \%{ }^{4}$.

Observamos cinco pacientes com cariótipo normal, dois foram submetidos à terapia de indução (casos 13 e 19). 0 paciente 13 
Pelloso Laf etal.

não atingiu $R C$ mesmo após três tentativas de indução, e o outro (caso 19) foi a óbito na fase de indução. Os outros três pacientes eram idosos (75, 81 e 84 anos) e devido a co-morbidades foi optado por terapia de suporte. $O$ fato de nessa amostragem os pacientes com cariótipo normal terem tido esse comportamento sem história prévia de doença hematológica levanta questões acerca de outros fenômenos biológicos envolvidos na doença. Pacientes com cariótipo normal ficam estratificados numa escala intermediária apresentando uma taxa de RC de $63 \%$ segundo o estudo $\mathrm{MRCI}$, e desses, $17 \%$ dos casos têm doença refratária e $20 \%$ vão a óbito na indução ${ }^{18}$.

Este trabalho permitiu evidenciar a importância do cariótipo na definição dos grupos de risco e a necessidade desse exame para 0 tratamento de LMA.

\section{Conclusões}

A taxa de cariótipo alterados em LMA (80\%) está dentro dos valores relatados pela literatura (65\%-95\%). Ficou nítida a diferença entre a taxa de RC do grupo favorável $x$ desfavorável, confirmando a importância do cariótipo.

\section{SUMMARY}

KaRYotype IN ACUTE MYELOID LEUKEMIA: IMPORTANCE AND TYPE OF ABERRATIONS IN 30 PATIENTS AT DIAGNOSIS.

INTRODUCTION. Cytogenetics in AML at diagnosis is a well defined prognostic tool. Objective: The authors analized karyotype(KT) and clinical data of newly diagnosed AML patients.

METHODS. Thirty patients were studied, 16 male and I 4 female. Age ranged from 19 to 84 years. Diagnostic criteria were based on WHO classification, immunophenotyping and $G$ banding cytogenetics. They were treated according to standard protocol (daunorrubicin and cytarabine $-3+7)$ and those who had Acute Promyelocytic Leukemia additionally receivedATRA.

RESULTS. KT success rate was $84 \%$. According to KT patients were divided into 4 groups: favourable prognosis (FP) (6) $(t(8 ; 2 I), t(15 ; I 7))$; intermediary prognosis (IP) (7) (four normal karyotypes, $+8, t(I ; 2)$ and del $18(q))$; unfavourable prognosis (UP); and 3 secondary AML; two evolving from prior Mylelodysplastic Syndrome and one presenting as an initial blast crisis of chronic myeloid leukemia. The median age of FP was 23 years while UP was 60 years $(p<0.003)$. In the FP, 5/6 (83\%) achieved complete remission $(C R)$ while only $I / 7$ (20\%)in the IP and I/8 (I2,5\%) in the UP. There was a tendency of higher leukocyte count in the unfavourable group.

CONCLUSIONS. The rate of karyotype aberrations in $A M L$ was $80 \%$ and in accordance to literature data (65-95\%). There was a clear difference in CR rates between favourable and unfavourable prognosis group. [Rev Assoc Bras 2003; 49(2): 150-5]

KEY WORDS: Acute Myeloid Leukemia. Karyotype.

\section{REFERÊNCIAS}

I. Look T. Oncogenic transcript factors in the human acute leukemias. Science 1997; 278: 1059-64.

2. Campana D, Behm FG. Immunophenotyping of leukemia. J Immunol Methods 2000; 243: 59-75.

3. Bahia DMM, Yamamoto M., Chauffaille Mde L, Kimura EY, Bordin JO, Filgueiras MA et al. Aberrant phenotypes in acute myeloid leukemia: a high frequency and clinical significance. Haematologica 200 I; 86:80I-6.

4. Mrózek K, Heinonen K, De la Chapelle A, Bloomfield C. Clinical significance of cytogenetics on acute myeloid leukemia. Semin Oncol 1997; 24: 17-31.

5. Grimwade D, Walker $H$, Oliver $F$, Wheatley K, Harrison C, Harrison G et al. The importance of diagnostic cytogenetics on outcome in AML; analysis of 1612 patients entered into the MRC AML 0 trial. Blood 1998; 92:2322-33.

6. Harris NL, Jaffe ES, Diebold J, Flandrin G, Muller-Hermalink HK, Vardiman J et al. World Health Organization classification of neoplastic diseases of the hematopoietic and lymphoid tissues: Report of the Clinical Advisory Comittee Meeting- Airlie
House, Virginia, 1997. J Clin Oncol 1999; 1 7:3835-49.

7. Jaffe ES, Harris NL, Stein H, Vardiman JW, editors In: World Health Organization Classifications of Tumours. Pathology and genetics of tumours of haematopoietic and lymphoid tissues. Lyon: IARC Press; 200I.

8. Bennett J, Catovsky D, Daniel MT, Flandrin G, Galton DA, Gralnick HRet al. Proposals for the classification of the acute leukemias. $\mathrm{Br} J$ Haematol 1976; 33:45 I-8.

9. Bennett], Catovsky D, Daniel MT, Flandrin G, Galton DA, Gralnick HR et al. The morphological classification of acute lymphoblastic leukemia: concordance among observers and clinical correlations. Br J Haematol 198I;47: 553-6I.

I0. Bennett J, Catovsky D, Daniel MT, Flandrin G, Galton DA, Gralnick HR et al. Proposed revised criteria for the classification of acute myeloid leukemia: a report of the FrenchAmerican-British Cooperative Group. Ann Intern Med 1985; 103:620-5.

I I. Bennett], Catovsky D, Daniel MT, Flandrin G, Galton DA, Gralnick HR et al. Proposal for the classification of minimally differentiated acute myeloid leukemia (AML-MO). Br J Haematol I991;78:325-9.

12. Rothe G, Schimirtz G. For the working group on flow cytometru and image analysis. Members of the editorial commitee Adorf D. Berlage S. Gramatzki. Consensus protocol for the flow cytometric immunophenotyping of haematopoietic malignancies. Leukemia 1996; 10:877-95.

13. Chauffaille MLLF, Figueiredo MS, Beltrani R, Antunes SV, Yamamoto M, Kerbauy J. Acute promyelocytic leukemia: the study of $\mathrm{t}(15 ; 17)$ translocation by fluorescent in situ hybridization, reverse transcriptase-polymearse chain reaction and cytogentic techniques. Braz J Med Biol Res 200 I ; 34:735-43.

14. ISCN: Ann International System for Human Cytogenetics Nomenclature. Mitelman F. Basel: Karger; 1995.

15. Cheson BD, Cassileth PA, Head DR, Schiffer CA, Bennett JM, Gale RP et al. Report of the National Cancer Institute- sponsored workshop on definitions of diagnosis and response in acute myeloid leukemia. J Clin Oncol 1990; 8:8I3-9.

16. Slovak ML, Kopecky KJ, Cassileth D, Harrington DH, Thiel KS, Mohamed A et al. Karyotypic analysis predicts outcome of preremission and postremission therapy in adult acute myeloid leukemia: a Southwest Oncology Group/ Eastern Cooperative Oncology group study. Blood 2000; 96:4075-83.

17. Lancet JE, Willman CL, Bennett J.M. Acute myelogenous Leukemia and aging: clinical 
interactions. Hematol Oncol Clin North Am 2000; 14:25I-67.

18. Grimwade D, Walker H, Harrison G, Oliver F, Chatters S, Harrison CJ. The predictive value of hierarchial classification in older adults with acute myeloid leukemia: analysis of 1065 patients entered into the United Kingdom Research Council AML I I trial. Blood 200 I; 98: I3 I 2-20.

19. Pelloso LAF, Baiocchi OCG, Chauffaille MLLF, Yamamoto M, Hungria VTM, Bordin JO. Magakaryocytic blastic crisis as a first presentation of chronic myeloid leukemia. Eur J Haematol 69:58-6I,2002.
20. Dastugue N, Payen C, Lafage-Pochitalof M, Bernard P, Leroux D, Stoppa AM. Prognostic significance of karyotype in de novo adult acute myeloid leukemia. Leukemia I 995; 9: I 49 I-8.

21. Joventino LP, StockW, Lane NJ, Daly KM, Mick $R$, Le Beau MM et al. Certain HLA antigens are associated with specific morphologic and cytogenetic subsets of acute myeloid leukemia. Leukemia I 995; 9:433-39.

22. Harada H, Harada Y, Eguchi M, Dohy H, Kamada N. Characterization of acute leukemia with $\mathrm{t}(4 ; 12)$. Leuk Lymphoma 1997; 25:47-53.

23. Sainty D, Arnoulet C, Mozziconacci MJ, De Pina JJ, Garnotel E, Lafage-Pochitaloff M. $\mathrm{t}(4 ; \mid 2)(\mathrm{q}|\mathrm{I} ; \mathrm{p}| 3)$ in a CD7 negative acute myeloid leukaemia. $\mathrm{Br} J$ Haematol 1997; 96:210-3.

24. Chauffaille MLLF, Fermino FA, Pelloso LAF, Silva MRR, Bordin JO, Yamamoto M. $\mathrm{t}(4 ; \mid 2)(\mathrm{qI} ; \mathrm{pl})$ : a rare chromosomal translocation in acute myeloid leukemia. Leuk Res 27: 363-366,2003.

Artigo recebido: 30/04/02 Aceito para publicação: 29/07/02 